\title{
Duplicate Suppression for Efficient Floating Car Data Collection in Heterogeneous LTE-DSRC Vehicular Networks
}

\author{
Ion Turcanu ${ }^{\mathrm{a}, \mathrm{b}}$, Florian Klingler ${ }^{\mathrm{b}}$, Christoph Sommer $^{\mathrm{b}}$, Andrea Baiocchi ${ }^{\mathrm{a}}$, Falko Dressler $^{\mathrm{b}}$ \\ ${ }^{a}$ Dept. of Information Engineering, Electronics and Telecommunications \\ Sapienza University of Rome, Italy \\ ${ }^{b}$ Heinz Nixdorf Institute and Dept. of Computer Science \\ Paderborn University, Germany
}

\begin{abstract}
Collecting data from a large number of agents scattered over a region of interest is becoming an increasingly appealing paradigm to feed big data archives that lay the ground for a vast array of applications. Vehicular Floating Car Data (FCD) collection is a major representative of this paradigm. Massive data collection from floating vehicles is the key to Intelligent Transportation Systems. We address the design and performance evaluation of a data collection protocol for the use case of periodic data collection. We target robustness, optimizing the amount of data and the value of the collection period, keeping in mind the goals of autonomous node operation and minimal coordination effort. From a system point of view, we believe that best solutions should jointly exploit the Long Term Evolution (LTE) cellular access network and the Dedicated Short-Range Communication (DSRC) based Vehicular Ad Hoc Network (VANET). Through a detailed comparative analysis, we show that such a hybrid approach offers superior performance, especially as for offloading the cellular radio access. A lightweight signaling procedure is designed, based on the DSRC VANET, which is able to avoid most of the duplicated data records, even if a distributed operation approach is pursued. The impact of the proposed protocol on the VANET load is evaluated and proved to be quite small, so that it does not interfere with other VANET-specific messages.
\end{abstract}

\section{Introduction}

Collecting massive data from a vast set of agents scattered over a Region of Interest (ROI) is one of the key features of next generation cellular networks and of a number of increasingly popular applications. While having agents equipped with possibly multiple sensors gathering data that are periodically reported to some central facility is a well-established and widely investigated paradigm, there are evolving features that are undermining currently available solutions.

Two major issues are: (i) the steeply growing amount of data that is made available for collection, providing a potential boost of new applications; and (ii) the unprecedented number of individual terminals that could access the network concurrently and quite often. This last point marks a definite break with the paradigm of few broadband users that has lead the rush to higher network capacities for broadband applications. As a matter of example, it has been observed $[1,2,3,4]$ that Long Term Evolution (LTE) is definitely inefficient when accommodating a large population of agents that need to send limited amount of

Email addresses: ion.turcanu@uniroma1.it (Ion Turcanu), klingler@ccs-labs.org (Florian Klingler), sommer@ccs-labs.org (Christoph Sommer), andrea.baiocchi@uniroma1.it (Andrea Baiocchi), dressler@ccs-labs.org (Falko Dressler) data periodically. The main reason is the heavy procedural overhead associated with obtaining and configuring radio resources for carrying data. Those procedures are warranted when a large amount of data is to be transferred, but they become an unsustainable burden for intermittent sources that need to send relatively small data chunks.

Specifically, we address the vehicular networking environment [5], where agents are vehicles equipped with wireless communication capabilities. This is an especially interesting case for several reasons:

- Automotive applications have witnessed rapid growth over the last years and are on the brink of an explosive spread and impact;

- The density of vehicles in urban areas and the huge amount of data collected by on-board sensors make a major case of big data collection over time and space; and

- Vehicles have a number of features that suggest specific directions to be pursued for an effective data collection: they can be connected, they have no severe energy constraints (on the communication equipment), they can afford a relevant amount of processing and memory space [6].

Currently, the main technologies able to support automotive applications are LTE and Dedicated Short-Range 


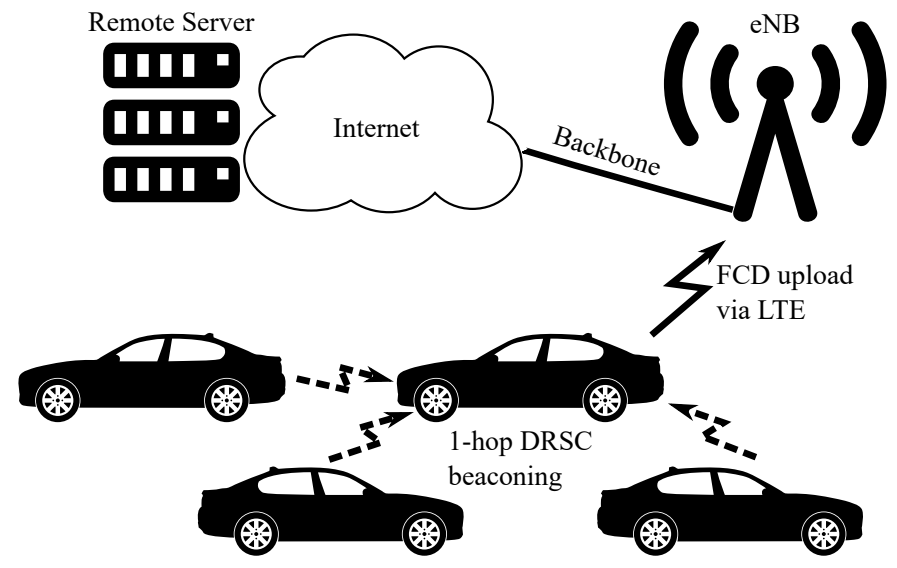

Figure 1: FCD collection scenario.

Communication (DSRC). LTE has been identified as a good candidate technology for supporting non-safety applications [7], like urban sensing and traffic efficiency. The reason is that LTE offers high throughput, promises high penetration rate, and has the advantage of being already widely deployed. However, this technology has several drawbacks. First of all, it operates in a licensed spectrum, meaning that its performance and availability is highly dependent on the mobile and network operators. Also, in high density urban scenarios the periodic data transmissions from many vehicles can use a significant part of the LTE channels, possibly degrading the normal operation of traditional applications. In order to support the increasing amount of data traffic, LTE needs further upgrades, like decreasing the cell sizes, or adding more spectrum [8]. All these upgrades are not for free, requiring additional investments from the network operators. On the other hand, DSRC has been proposed as the main technology to be used for supporting vehicular safety applications, because it can guarantee low message transmission delays, required by such applications. It also operates on a dedicated spectrum, which is specifically assigned for Intelligent Transportation Systems (ITS). On the downside, DSRC is not yet widely available. In order to support centralized services and applications, additional gateways and hardware is needed, like Roadside Units. The deployment of such infrastructure is expensive [9].

In this work, we address Floating Car Data (FCD) collection in an urban environment by exploiting both vehicle connectivity through DSRC and cellular coverage (LTE). In our previous work [10], we proposed On-theFly Clustering (OFC), a distributed clustering algorithm that elects forwarder vehicles to be in charge of sending their own data plus their neighboring vehicles' information through LTE. The election of forwarders is run by means of a completely distributed procedure exploring intermittent 1-hop beacon message exchange through DSRC, as shown in Figure 1. A timer-based protocol allows a simple way to identify nodes that win the election. The timer is set to a level depending on the number of DSRC neighbors of the node and on the quality of the LTE channel of the node, as assessed by means of the Channel Quality Indicator (CQI), besides a randomization component. The tuning of the parameters used for the timer computation has been carried out in [10]. The beaconing background process, envisaged by the DSRC standard by means of the Cooperative Awareness Messages (CAMs) [11], populates a node local database, namely the Local Dynamic Map (LDM) [12]. The list of neighbors and the FCD to be reported for them, in case the node is selected as forwarder, are taken from the LDM.

The main points that are highlighted by means of an extensive performance analysis based on detailed simulations are as follows. Compared with a simple approach that uses only LTE to carry all data, sent individually by each vehicle node, the use of DSRC offers a substantial reduction of the load on the LTE access network, as we show by means of a state of the art protocol that exploits the DSRC connectivity parameter for electing cluster head vehicles. The load is further reduced with OFC; its forwarder election process combines the CQI in the LTE uplink with the DSRC connectivity. We then propose an extended version for the OFC algorithm, named OFC with Duplicate Suppression (OFCDS), that addresses the problem of a high number of generated duplicates through a more effective duplicate suppression algorithm by introducing more control messaging on DSRC. The price of offloading the LTE access network lies in an increase of the delay to transfer the data to the remote facility. Hence, there is a trade-off between delay and load of the cellular network. The information to be sent to the remote facility is periodically updated by the background beaconing process, which is the main load generator for the DSRC network. Overall, the load on DSRC due to beaconing amounts to few percent of the capacity, even when the duplicate suppression mechanism is used. Still, the additional load due to the control messages defined by our proposed protocol for coordinating the data collection through the LTE network amounts to a negligible overhead with respect to beaconing.

A major takeaway of the present work is a quantitative assessment of the benefit brought about by a cooperation between the Vehicular Ad Hoc Network (VANET) based on the DSRC technology and the cellular network to tackle the issue of massive spatio-temporal data collection. While 5G is expected to improve substantially the efficiency of M2M communications, it does not seem reasonable to give up exploiting the potential of the VANET, both as regards its support of safety messages (which can be deemed to have the highest priority) and to support signaling procedures to coordinate the nodes for an efficient usage of the cellular radio resources.

The rest of the paper is organized as follows. Section 2 offers a concise survey of the related literature. A background for the CQI in the LTE uplink is given in Section 3. An use case application relying on intermittently collected FCD, as well as a description of existing FCD collection algorithms are presented in Section 4. The details of the 
new proposed protocol are defined in Section 5. Then, an introduction of the simulation scenario and an extensive performance evaluation is presented in Section 7. Final remarks are given in Section 8.

\section{Related Work}

LTE has been identified as a potential access technology able to support vehicular communications [7, 13, 14]. There are several reasons why LTE is suitable. First of all, it has the benefit of an already pre-deployed infrastructure, which offers wide area coverage and supports high mobility. Secondly, the market penetration rate of LTE is expected to be higher compared to other communication technologies, since the LTE technology is already integrated in common user devices, like smartphones, tablets, and smartwatches. Moreover, many vehicular applications can migrate to these devices.

Araniti et al. [7] provide an extensive survey on the state of the art of LTE and its capability to support vehicular applications. Mangel et al. [13] analyze the usability of LTE for vehicular safety communication at intersections, comparing them with DSRC. They conclude that even if LTE seems able to support periodic delivery of beacon messages, its performance in terms of awareness update rate and latency is inferior with respect to DSRC. On the other hand, the latency and reliability requirements are not so strict for non-safety applications. Yet, the information generated by a high number of vehicles can heavily load the uplink channel, preventing the normal operation of traditional human-to-human traffic. Ide et al. [14] propose a channel sensitive probabilistic transmission scheme in order to reduce the LTE channel load. Their algorithm reduces the number of forwarders, but does not guarantee an exhaustive collection of data.

DSRC has been proposed as the main technology for Inter-Vehicle Communication (IVC). The primary motivation is to ensure safety on the roads by enabling Vehicleto-Vehicle communication and cooperative awareness. The latter is usually obtained through periodic exchange of beacon messages. Beacons contain vehicles' position, velocity, direction of travel, and other basic information. They are periodically broadcast from either vehicle to its neighbors, so that every vehicle at every time instant has an updated list of its one-hop neighbors. These messages are referred to as CAMs [11] or Basic Safety Messages (BSMs) [15].

Among the advantages that DSRC offers we can identify low message delays, decentralized architecture, and localized network load. However, to support non-safety applications, DSRC needs additional hardware and infrastructure deployment, like Roadside Units. Moreover, the technology currently is not yet widely deployed, meaning that at least in the initial stage DSRC needs to be supported by other existing communication technologies.

To cope with the limitations that both LTE and DSRC have, the research community is shifting towards heterogeneous vehicular networking approaches [16, 17, 18, 19,
$20,21,22]$. The idea is to deploy both technologies to vehicles and road, and to exploit the benefits from each technology. A common paradigm is to use the cooperative awareness enabled by DSRC to create clusters of vehicles having common features (e.g., proximity, travel direction, speed, connectivity). A complete taxonomy on clustering in vehicular networks is proposed by Bali et al. [23]. There a comprehensive analysis of existing proposals in literature is provided, as well as a detailed discussion for each category of clustering, including challenges and future directions.

Non-safety applications usually require periodic collection of data from vehicles inside a target area. Various applications have different requirements in terms of accuracy of the reported information. For instance, Ide et al. [16] focus on a traffic forecast application where neighboring vehicles have similar information. Based on this assumption, elected forwarding vehicles perform local aggregation and compression before sending the information to the remote server via LTE. The upper bound of the amount of compressed data is modeled as a square root function of the number of uncompressed data units. However, in many non-safety applications the information cannot be compressed, meaning that data from every single vehicle must be gathered. In this case, which is also the case that we consider in this paper, the aggregation consists of concatenating the payloads gathered from the DSRC neighboring vehicles.

The target application has a strong impact on the decision of what parameters to consider in the clustering mechanism. Many applications aim at obtaining cluster stability, meaning that the vehicles' position, speed, and driving direction are the most critical parameters. Other applications focus on minimizing the LTE channel utilization while periodically collecting data from vehicles. In this case DSRC connectivity becomes predominant, since the main objective is to collect data from the whole vehicle network, while minimizing the number of forwarders and maximizing the local aggregation.

Stanica et al. [17] identify this as to be equivalent to the Minimum Dominating Set problem in graph theory, known to be NP-complete. They propose three heuristics for the election of Cluster Head vehicles in a heterogeneous LTE/DSRC vehicular network: Degree-Based $(D B)$, Degree-Based with Confirmation $(D B-C)$, and ReservationBased $(R B)$. $D B$ basically uses the safety beacons exchanged over the DSRC network to compute the number of neighbors for each vehicle. This information is used by the forwarded election mechanism, in which a vehicle becomes a forwarder with a probability equal to $k / D$, where $D$ is the number of neighbors and $k$ is a parameter for the trade-off between coverage and offloading gain. Although it is very simple, this algorithm does not provide any guarantee on the coverage of the entire area. $D B-C$ copes with this issue by extending the previous approach with a simple confirmation mechanism in order to obtain the total coverage. The idea is that whenever a vehicle chooses to be a forwarder it informs its neighbors by broad- 
casting a notification message. If during a collection period a vehicle does not become a forwarder and does not receive any notification message, it deems to be disconnected and sends its own information via LTE. With $R B$ each vehicle, at the beginning of every collection period, selects a transmission slot among $N_{\mathrm{s}}$ available and waits for its slot to transmit. Whenever a vehicle transmits the data on LTE, it becomes dominator and informs its neighbors, who cancel their waiting times and become dominated. A crucial point in this mechanism is the choice of $N_{\mathrm{s}}$.

These algorithms are evaluated in terms of system gain, defined as the fraction of vehicles that do not have to access the cellular infrastructure when data is offloaded through DSRC communication. However, this metric does not directly measure the utilization of the LTE channel. In this article we are actually focusing on measuring the Resource Block (RB) utilization in the LTE network. Moreover, Stanica et al. [17] assume a simple unit disk model for IVC connectivity, where two vehicles can communicate whenever their distance is below a threshold $R$, which is a nonrealistic assumption. Also, most of the heuristics presented above are trying to minimize the number of forwarders by relying only on the DSRC connectivity parameter.

In this article we show that considering the CQI [24] in the LTE uplink and some jitter in addition to the DSRC connectivity, and properly combining them in the forwarder selection process can yield significant offloading of LTE. Also, by introducing a more efficient duplicate suppression algorithm, we are able to keep the RB utilization level lower with respect to existing solutions, even when increasing the frequency of the collected information.

\section{LTE Channel Quality Indicator}

One of the key features of LTE is the possibility of selecting the downlink/uplink transmission configuration and related parameters depending on the current channel condition, including the interference situation [25]. The instantaneous channel quality, namely CQI, is provided periodically or aperiodically by the terminals to the eNodeB. The eNodeB makes up decisions on resource allocation based on the terminal CQI information. Periodic CQI reports can be transmitted on the Physical Uplink Control Channel (PUCCH) or Physical Uplink Shared Channel (PUSCH), while aperiodic reports can be transmitted only on PUSCH.

In LTE, CQI provides quantized indication of the highest modulation and coding scheme that, if used by the eNodeB, lets the User Equipment (UE) demodulate and decode the transmitted downlink data with a maximum block error rate of $10 \%$. However, the CQI is only a recommendation, meaning that the eNodeB does not need to necessarily use it. The reason is that the eNodeB has to consider also other information when allocating resources. For instance, if the UE needs to transmit only a small amount of data, then there is no need to select a very high data rate, because a small number of $\mathrm{RBs}$ with robust modulation is sufficient. There are 15 different CQI values, ranging from 1 to 15 . The higher the CQI value reported by the UE, the richer the modulation scheme (from QPSK to $64 \mathrm{QAM}$ ) and the bigger the coding rate used by the eNodeB to improve the efficiency as much as possible.

There is no explicit description in the standard documents of the mechanism by which the CQI is calculated, but it is known that the Signal to Noise Ratio (SNR) and/or Signal to Interference plus Noise Ratio (SINR) factors play important roles in the CQI computation. How these factors should be used and whether there are any other factors that should be involved is not well defined. Our estimation of the CQI is based on the work of Virdis et al. [26], which uses a mapping table of measured block errors to determine the CQI based on a given SINR value. ${ }^{1}$ The SINR is computed as

$$
\mathrm{SINR}=\frac{P_{\mathrm{s}}}{\sum_{i} P_{i}+N}
$$

where $P_{\mathrm{s}}$ is the power received from the serving eNodeB, $P_{i}$ is the power received from the interfering eNodeB $i$, while $N$ is background Gaussian noise. The received power $P$ is computed as

$$
P[\mathrm{dBm}]=P^{\mathrm{tx}}[\mathrm{dBm}]-L_{\mathrm{P}}[\mathrm{dB}]-L_{\mathrm{S}}[\mathrm{dB}]-L_{\mathrm{F}}[\mathrm{dB}]
$$

where $P^{\mathrm{tx}}$ is the transmit power, $L_{\mathrm{P}}$ is the path loss [27], while $L_{\mathrm{S}}$ and $L_{\mathrm{F}}$ represent the attenuation due to slow and fast fading, respectively.

Since the main idea behind the clustering algorithms in heterogeneous vehicular networks is to properly choose the forwarding vehicles, we believe that the CQI has to be used in the selection process. Not using the CQI parameter can lead to the election of forwarding vehicles having a poor CQI. For such forwarders, which have to send a high amount of aggregated information via LTE, the eNodeB wastes plenty of resources, leading to an inefficient utilization of the LTE channel. The waste of resources comes from the modulation and coding scheme that the eNodeB chooses according to the reported CQI. This means that a vehicle with a lower CQI value encodes much less information in one RB than a vehicle having a higher CQI.

\section{Application and algorithms}

We first present a sample application used in our study, showing a simple LTE-based data collection algorithm. Then we describe the OFC algorithm proposed in [10], that uses both LTE and DSRC technologies to collect data in a heterogeneous vehicular network. Finally, we show how OFC can be turned into a baseline state of the art distributed clustering algorithm that is basing its forwarder selection procedure on the current number of DSRC neighbors only.

\footnotetext{
${ }^{1}$ http://github.com/inet-framework/simulte
} 


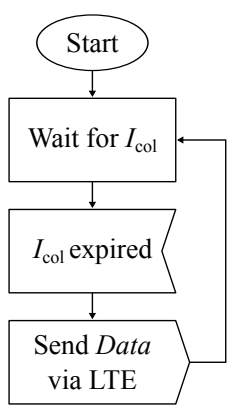

Figure 2: PureLTE data collection algorithm.

\subsection{LTE-based traffic monitoring application}

We consider a traffic monitoring system as use case example for our study, but any other application that needs periodic exhaustive collected information is relevant. We assume that every vehicle inside the target area has LTE communication technology available on board. The application itself consists in periodically reporting FCD messages via LTE to the traffic monitoring system server. The updating frequency, which is common to all vehicles, is decided by the traffic monitoring system and is set up in the collection interval parameter $\left(I_{\text {col }}\right)$ by every vehicle (i.e., when the application starts, it can immediately send a request to the remote server via LTE asking for the desired reporting frequency).

A simple algorithm that periodically collects FCD messages in such a scenario is presented in Figure 2. We will further refer to this approach as PureLTE. Basically, whenever the application starts, it periodically schedules a time-out event, named $I_{\text {out }}$, equal to the collection interval parameter. When the time-out expires, the application sends a Data message via LTE to the traffic monitoring system server containing updated information about the vehicle itself. Notice that a Data message can contain one or more FCD messages. In this particular case Data consists of only one FCD message created by the transmitting vehicle itself, since no IVC communication is present. The transmissions are not synchronized among different vehicles. The only common information that must be known to all vehicles is the parameter $I_{\mathrm{col}}$.

Although this approach is very simple, it implies that every vehicle has to periodically report its FCD, which can introduce a high load over the LTE channels, especially in the case of urban scenarios with high vehicle density [28]. Considering that many different vehicular applications, as well as all regular LTE traffic, will have to share the same limited LTE bandwidth provided by the mobile and network operators, this issue becomes even more critical.

\subsection{OFC algorithm}

The FCD collection application assumes each vehicle maintains a Local Data Base (LDB) where the relevant information about the vehicle itself and about its current neighbors is stored. A background exchange of one-hop

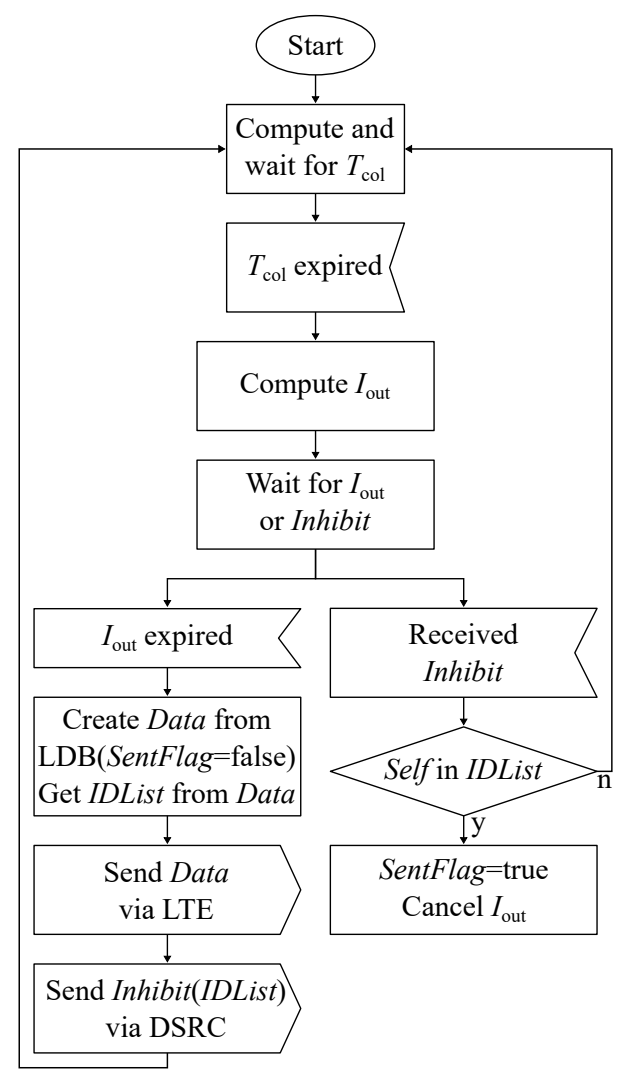

Figure 3: OFC data collection algorithm.

messages on DSRC keeps the LDBs up to date. When the time comes for sending a report, the elected forwarding vehicle reads its current LDB content and sends it to the remote server. An example of such a process is already envisaged explicitly by ETSI standards, where the CAMs exchanged among neighboring vehicles and the Local Dynamic Map [12] database are defined to maintain vehicle awareness of the surrounding vehicular traffic environment. We do not pursue the details of the LDB maintenance further, since this has been widely investigated in the literature (e.g., see [29][30]).

The main idea behind OFC is to allow only a subset of vehicles, named forwarders, to report via LTE their own, as well as their one-hop neighbors' Floating Car Data. These forwarders are dynamically selected during every collection interval. The selection process itself is based on synchronized selection phases and takes into account the current number of DSRC neighbors, the CQI in the LTE uplink information, and a uniformly distributed random jitter. OFC operation is highlighted in Figure 3. Unlike the PureLTE approach, where no synchronization is needed since no IVC is present, with OFC the time instance when the collection interval starts must be the same for all vehicles. Although the forwarder selection mechanism is performed locally, it has to start at the same point in time for all vehicles, since the considered parameters have to refer to the same time instance. Hence, every vehicle is periodically computing 
the next collection interval according to

$$
T_{\text {col }}=T_{\text {cur }}-\left(T_{\text {cur }} \bmod I_{\text {col }}\right)+I_{\text {col }}
$$

where $T_{\text {col }}$ is the point in time when the collection interval starts, $T_{\text {cur }}$ is the current time instance (i.e., we assume every vehicle has a GPS on board which can provide the current time) and $I_{\text {col }}$ represents the collection interval span.

Upon collection interval starting, every vehicle computes its own sending time $T_{\text {send }}$ according to

$$
T_{\text {send }}=T_{\text {col }}+I_{\text {out }}
$$

where the time-out interval $I_{\text {out }}$ is given by

$$
I_{\mathrm{out}}=I_{\mathrm{col}}(\alpha X+\beta Y+\gamma Z)
$$

Here $\alpha, \beta$, and $\gamma$ are non-negative weights chosen so as that $\alpha+\beta+\gamma=1$, and $\alpha, \beta, \gamma \in[0,1] . X, Y$, and $Z$ represent the DSRC connectivity, the CQI in the LTE uplink, and the jitter respectively and are computed as

$$
\begin{gathered}
X=1-\frac{N_{\text {cur }}}{N_{\max }} \\
Y=1-\frac{Q_{\text {cur }}}{Q_{\max }} \\
Z=\mathcal{U}(0,1)
\end{gathered}
$$

where $N_{\text {cur }}$ and $Q_{\text {cur }}$ represent the current number of onehop DSRC neighbors and the current CQI in the LTE uplink of a generic vehicle (in case of subband-level CQI reporting, the average value over all subbands is considered), while $N_{\max }$ and $Q_{\max }$ are the corresponding maximum values. Notice that $Q_{\max }$ refers to the maximum CQI index, which is globally known to all vehicles, while $N_{\max }$ is locally computed by every vehicle. In particular, $N_{\text {cur }}$ is included in the beacon exchange process, meaning that every vehicle knows the number of neighbors for each one of its one-hop DSRC neighbors. At this point a vehicle can compute $N_{\text {max }}$ by finding the maximum $N_{\text {cur }}$ value among all its neighbors.

In [10] we analyzed the impact that each of the three considered factors has by studying the influence of the parameters $\alpha, \beta$, and $\gamma$ on the LTE resources utilization. According to Equation (5), vehicles having a higher number of DSRC neighbors and a better CQI in the LTE uplink are scheduled for transmission first. Vehicles whose time-out expires become forwarders and prepare their Data message to be sent to the traffic monitoring system by reading their LDB. Immediately after sending the Data message via LTE, a forwarder has to inform its neighbors by broadcasting an Inhibit message over the DSRC network, containing the identifiers of all vehicles whose FCD was enclosed in Data. If a vehicle waiting for its time-out to expire receives an Inhibit message, it checks whether its identifier is present. If this is true, then it immediately cancels the time-out $I_{\text {out }}$, aborting its scheduled transmission. According to this

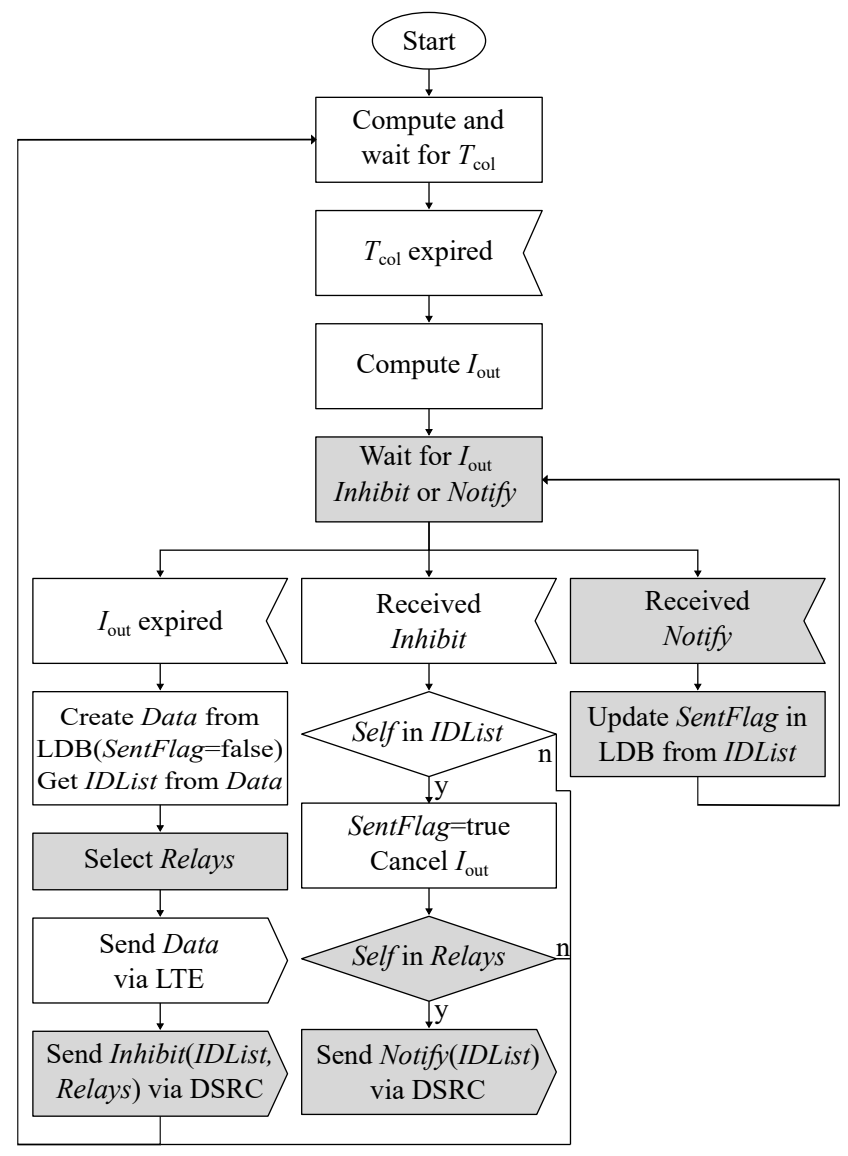

Figure 4: OFCDS data collection algorithm.

approach, once a vehicle becomes a forwarder, all its 1-hop DSRC neighbors are inhibited.

Notice that an inhibited vehicle can be in the transmission range of more than one forwarder, meaning that multiple copies of the same FCD message can be sent to the server, increasing the LTE channel utilization. OFC has a duplicate suppression mechanism that takes advantage of the already existing beacon exchange process. In particular, the beacon messages sent in background are extended with a flag, named SentFlag. At the beginning of each collection cycle, vehicles set their SentFlag to FALSE. As soon as a vehicle $A$ receives an inhibition message from a neighbor, announcing that the neighboring vehicle has reported $A$ 's FCD to the remote server, $A$ turns its flag to TRUE. Whenever a vehicle node updates the application information by sending a message to its neighbors, it includes the current value of its SentFlag. As a consequence, updates of the application data sent by the inhibited vehicle $A$ every $I_{\mathrm{LDB}}$ seconds carry the flag set to TRUE and cause the relevant information to be updated in the LDBs of $A$ 's neighbor vehicle nodes. If any of those neighbors report their Data to the remote server, they will exclude $A$ 's FCD.

\subsection{Baseline algorithm}

Current state of the art solutions consider the DSRC connectivity as the main parameter in the forwarder elec- 
tion mechanism $[17,18]$. These are usually heuristics for finding approximations to the Minimum Dominating Set problem, that aim at maximizing the offloading level by minimizing the number of forwarders.

OFC structure has the flexibility to be easily turned into such algorithm, that we will further refer to as Baseline, by using only the number of DSRC neighbors as the main parameter, while not using the CQI in the LTE uplink at all. This can be done by properly setting the values for the weighting factors $\alpha, \beta$, and $\gamma$. For instance, by setting $\beta=0$ we end up having a heuristic which is minimizing the number of forwarders by selecting those vehicles with the highest number of DSRC neighbors. Notice that we still keep $\gamma=0.2$ (i.e., jitter) to reduce simultaneous transmissions and obtain a fair comparison with the other considered solutions.

The features of the Baseline algorithm are similar to the $R B$ clustering mechanism proposed by Stanica et al. [17] and described in Section 2. According to this solution each vehicle transmits in a slot selected at the beginning of every collection interval, chosen among $N_{\mathrm{s}}$ available, only if no other neighboring vehicle transmitted its information first. However, since the authors do not specify the slot selection criterion, we can assume that with this heuristic a vehicle selects its own slot uniformly at random. In [10] we proved that a clustering algorithm that considers only the randomness factor in the forwarder selection process turns out to be sub-optimal. For this reason, in our implemented version of the Baseline algorithm we consider the number of DSRC neighbors by giving priority to those vehicles that have more DSRC neighbors.

\section{OFC with Duplicate Suppression}

The effectiveness of the SentFlag mechanism described in Section 4.2 depends on the ratio between the time interval $I_{\mathrm{LDB}}$ of the background application LDB periodic update and the data collection time interval $I_{\text {col }}$ : the smaller $I_{\mathrm{LDB}} / I_{\mathrm{col}}$, the more effective the SentFlag mechanism. However, for applications that need frequent information updates from the vehicular network, this mechanism can be less effective in preventing the transmission of duplicate messages on LTE, causing a higher resources utilization. For this reason, we extend here the OFC algorithm with a new duplicate suppression scheme that does not depend on the $I_{\mathrm{LDB}} / I_{\mathrm{col}}$ ratio. The main idea behind this new approach, named OFCDS, is to immediately disseminate the IDs of all 1-hop DSRC neighbors whose information is being sent on LTE by an elected forwarder to all its 2-hop DSRC neighbors.

OFCDS operation is displayed in Figure 4. In particular, the new parts of the extended algorithm, as well as the modified parts from Figure 3, are represented by the gray blocks. In Algorithm 1 we present the pseudo-code of our proposed solution. Notice that the inhibition mechanism in OFCDS is similar to OFC. In particular, once a vehicle is elected as forwarder, it immediately broadcasts

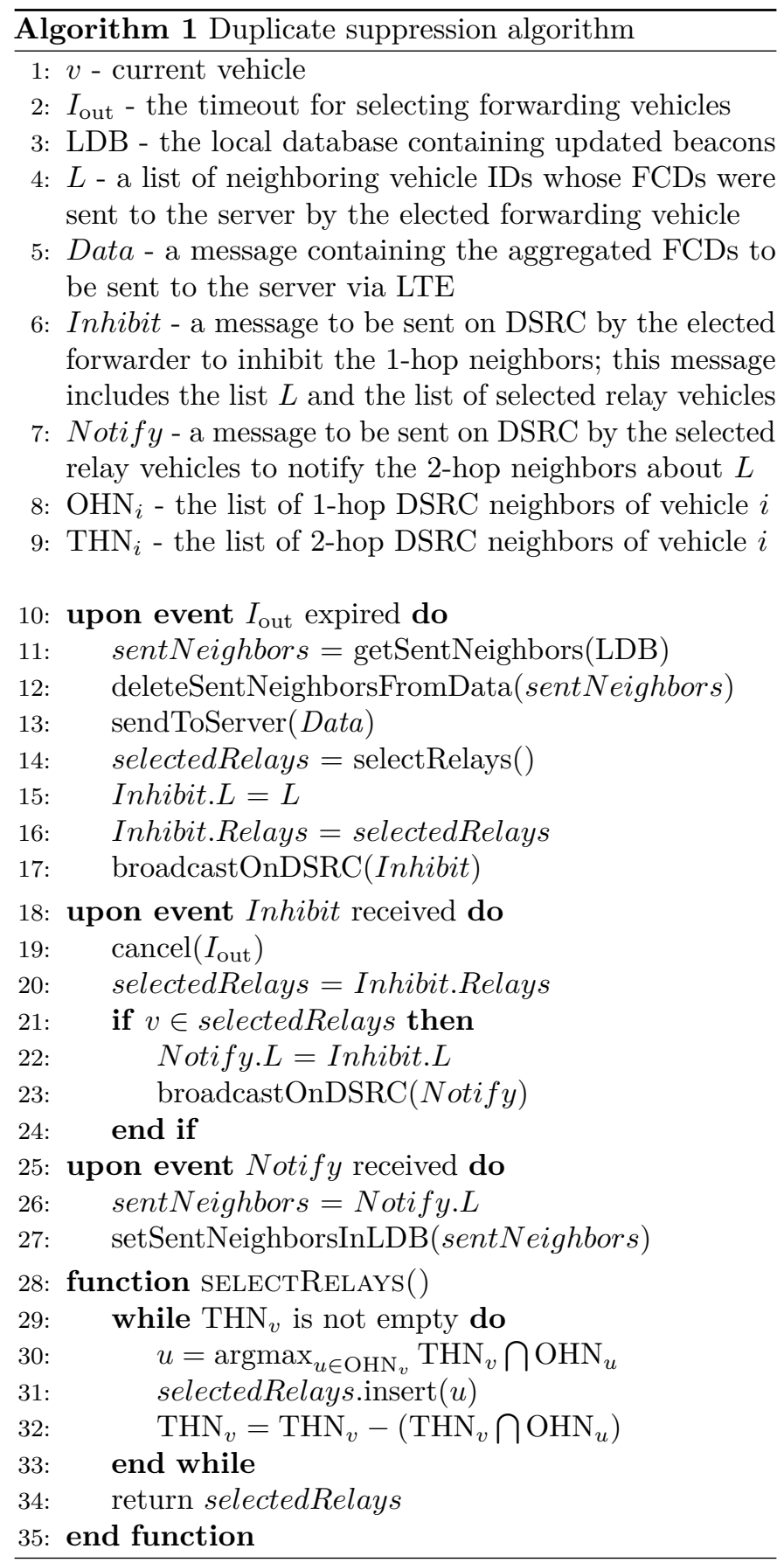

an Inhibit message containing the ID list $L$ of its 1-hop DSRC neighbors whose information was included in the Data message. The main difference is in the behavior of the vehicles that receive the Inhibit message. Unlike OFC, where the inhibited vehicles wait for the next beaconing opportunity to inform their neighbors about the fact that their information was already sent, in OFCDS the inhibited vehicles must disseminate as soon as possible to all their neighbors the full list $L$ received from the elected forwarder. They do this by broadcasting a Notify message including the list $L$, with a small random delay to avoid simultaneous transmissions. Notice that Inhibit is telling 


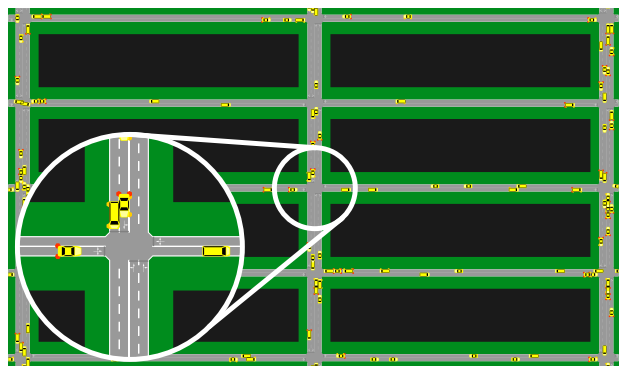

Figure 5: Part of the simulation scenario.

which vehicles should cancel their $I_{\text {out }}$ timers, while Notify is only informing about the inhibited vehicles, so that other potential forwarders can exclude the corresponding FCDs from their Data messages.

However, to avoid the congestion of the DSRC channel, not all inhibited vehicles are broadcasting the Notify message. The idea is that the elected forwarders select a subset of their 1-hop DSRC neighbors to be in charge of sending such message. The relay selection procedure (selectRelays() function in Algorithm 1) consists in iteratively selecting a subset of 1-hop neighbors, such that all 2-hop neighboring vehicles are covered. This is possible only if all vehicles have 2-hop DSRC awareness. We achieve this by extending the beacon structure with the current ID list of 1-hop DSRC neighbors. Notice that while the standard beacon has a fixed constant size, the extended beacon size depends on the current number of DSRC neighbors.

\section{Simulation setup}

For evaluating the proposed algorithms we use Veins LTE [31], an LTE extension of the well-known open source vehicular network simulator Veins ${ }^{2}$ [32]. A realistic Manhattan grid scenario is considered for our simulations, created using real Manhattan downtown road and building dimensions (see Figure 5). Krauss vehicular mobility model is used, along with the random trips traffic flow origindestination model. Although the vehicular mobility is simulated over a larger area, we enclosed the observed region to a smaller target area to avoid border effects. Also, we use the free-space path loss $(\alpha=2)$ with obstacle shadowing [33] models for DSRC, and Urban Macro path loss [27] with Jakes multi-path fading models for LTE.

We assume LTE coverage is available inside the target area. All vehicles are equipped with DSRC and LTE wireless network interfaces, while the decision whether to send a packet on one interface or on another is taken at the application layer. Considering that most likely the mobile operators will dedicate only a small portion of bandwidth to vehicular applications, for our analysis we assume a bandwidth of $3 \mathrm{MHz}$ (15 available RBs). Since different traffic monitoring systems, but also other applications,

\footnotetext{
${ }^{2}$ http://veins.car 2 x.org
}

\begin{tabular}{lc}
\hline Parameter & Value \\
\hline Simulated area & $620 \mathrm{~m} \times 530 \mathrm{~m}$ \\
Average number of vehicles & 165 and 390 \\
Average density (veh/km/lane) & 11 and 26 \\
Simulation duration & $100 \mathrm{~s}$ \\
$I_{\text {col }}$ & $1,3,5,10,15$ and $20 \mathrm{~s}$ \\
Baseline $\alpha, \beta$, and $\gamma$ & $0.8,0,0.2$ \\
OFC and OFCDS $\alpha, \beta$, and $\gamma$ & $0.3,0.5,0.2$ \\
IVC technology & IEEE $802.11 \mathrm{p}$ \\
IVC maximal transmit power $P$ tx & 20 and $100 \mathrm{~mW}$ \\
DSRC beacon frequency $I_{\mathrm{LDB}}$ & 1 and $10 \mathrm{~Hz}$ \\
Beacon size & $400 \mathrm{~B}$ \\
Vehicle ID size $S$ & $6 \mathrm{~B}$ \\
Number of available RBs & 15 \\
LTE scheduler & MAXCI \\
UE transmission power & $26 \mathrm{dBm}$ \\
eNodeB transmission power & $45 \mathrm{dBm}$ \\
\hline
\end{tabular}

Table 1: Simulation parameters

might have particular requirements in terms of data reporting frequency, we analyze and compare the performance of the three considered solutions with respect to different collection intervals.

All simulations are run for $100 \mathrm{~s}$ preceded by $400 \mathrm{~s}$ of warmup time. Every simulation is repeated 25 times with independent random number seeds. The most relevant simulation parameters are displayed in Table 1.

\section{Performance Evaluation}

We propose and compare two different implementations of OFCDS. The first one, named OFCDS-Ideal, is an idealistic implementation of the algorithm, where the 2-hop DSRC awareness is assumed to be obtained without additional load on the DSRC communication channel. We assume here that the size of the list containing the IDs of the current neighboring vehicles, included in each beacon, is constant and has a negligible size with respect to the beacon length (e.g., $10 \mathrm{~B}$ for the ID list size with respect to $400 \mathrm{~B}$ for the beacon size). The second, named OFCDSReal, is a realistic implementation where the size of the ID list depends on the actual number of vehicle IDs that are included in this list. For instance, let $A$ be a generic vehicle and $N_{\mathrm{A}}$ its current number of DSRC neighbors. Then, the additional payload added to $A$ 's beacon is $N_{\mathrm{A}} S$, where $S$ is the size of a vehicle ID entry. Both OFCDS implementations are evaluated for two different vehicular densities and compared against OFC, PureLTE, and Baseline.

The aim of this evaluation is to measure the performance and the influence of the proposed solutions on both LTE and DSRC communication channels. The main evaluation metrics are defined in Table 2. On LTE, we are interested in measuring the RB utilization in uplink, defined as the average percentage of used RBs requested to transfer the FCDs of all vehicles roaming inside the area of interest. There are two main causes for the RB utilization: (i) the amount of transferred information on LTE, which depends on the actual payload coming from the number 


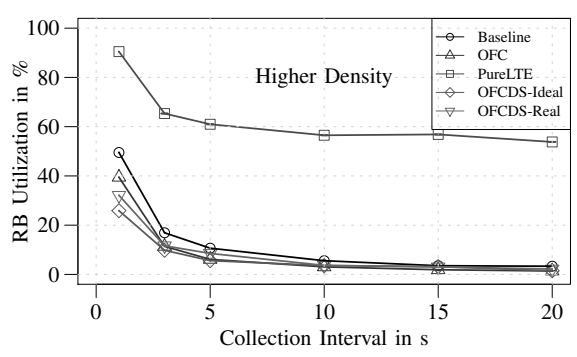

(a) $I_{\mathrm{LDB}}=1 \mathrm{~Hz}$ and $P^{\mathrm{tx}}=20 \mathrm{~mW}$.

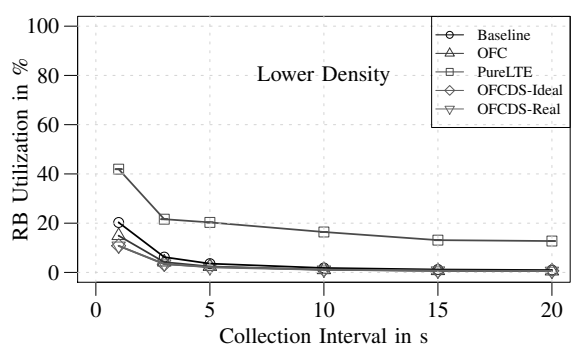

(b) $I_{\mathrm{LDB}}=1 \mathrm{~Hz}$ and $P^{\mathrm{tx}}=20 \mathrm{~mW}$.

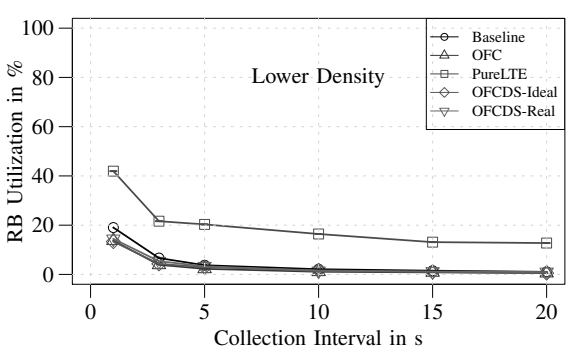

(c) $I_{\mathrm{LDB}}=10 \mathrm{~Hz}$ and $P^{\mathrm{tx}}=100 \mathrm{~mW}$.

Figure 6: The RB utilization as a function of the collection interval.

\begin{tabular}{ll}
\hline Metric & Definition \\
\hline RB Utilization & $\begin{array}{l}\text { Mean percentage of allocated RBs from the } \\
\text { total number of available RBs per each Trans- } \\
\text { mission Time Interval (TTI) }\end{array}$ \\
Duplicate Ratio & $\begin{array}{l}\text { Mean ratio of the number of duplicate mes- } \\
\text { sages to the total number of received mes- } \\
\text { sages in each collection interval }\end{array}$ \\
& $\begin{array}{l}\text { Time interval between the moment when the } \\
\text { Delay }\end{array}$ \\
$\begin{array}{l}\text { FCD message is generated and the time in- } \\
\text { remote server same message arrives at the }\end{array}$ \\
$\begin{array}{l}\text { Time difference between two consecutive } \\
\text { Time }\end{array}$ & $\begin{array}{l}\text { FCD message receptions at the server be- } \\
\text { longing to the same vehicle }\end{array}$ \\
Channel Busy & $\begin{array}{l}\text { Mean ration of the total time a vehicle senses } \\
\text { the DSRC channel busy to the total simula- } \\
\text { tion time }\end{array}$ \\
\hline
\end{tabular}

Table 2: Performance evaluation metrics

of FCD messages, and the overhead induced while transferring this information; (ii) the LTE channel quality of the transmitting vehicles. We tackle the second cause with our proposed solutions by considering the CQI in the LTE uplink in the forwarder election process. As for the first cause, we can act only on the generated overhead, which is coming from the network and transport layer headers, and the duplicate messages induced by the heterogeneous algorithms. We address these issues by significantly reducing the number of vehicles accessing the LTE network, that send aggregated FCD messages, and reducing the duplicate ratio, defined as the number of duplicate messages over the number of total received messages by the remote server.

Of course, reducing the RB utilization comes with a cost, which is paid in terms of information transferring delay, defined as the time interval between the moment when the FCD message is generated and the time instant when the same message arrives at the server. Moreover, we are interested in quantifying the variability of the arrived information, which is why we measure the inter-arrival time of the reported FCDs, defined as the time difference between two consecutive FCD message receptions belonging to the same vehicle being received at the remote server. At the same time, all the heterogeneous approaches introduce some load on the DSRC channel, which we measure as the average CBR experienced by each vehicle for the entire simulation period.

All these metrics are evaluated for different parameter configurations. In particular, we consider two vehicular densities, 11 and $26 \mathrm{veh} / \mathrm{km} /$ lane, with a lighter load on the DSRC channel: each vehicle's transmission power is set to $20 \mathrm{~mW}$ and the beaconing frequency to $1 \mathrm{~Hz}$. To evaluate the proposed algorithms under a higher DSRC load setup, we modified the low vehicular density scenario by increasing the transmission power to $100 \mathrm{~mW}$ and the beaconing frequency to $10 \mathrm{~Hz}$.

\subsection{Evaluation of RB utilization in LTE uplink}

The mean LTE RB utilization is depicted in Figure 6. We can notice that PureLTE is using the highest amount of RBs, independently from the considered collection interval and/or vehicular density, with a peak of $90 \%$ used RBs for a collection interval of $1 \mathrm{~s}$ and a higher vehicular density. This is an expected result, considering the fact that all vehicles roaming inside the area of interest are periodically accessing the LTE channel and requesting resources. On the other hand, for the same collection interval, all other algorithms that are exploiting the DSRC technology are significantly decreasing the LTE RB utilization, confirming the fact that the DSRC technology can help in decreasing the LTE channel utilization. Although for higher collection intervals the RB utilization is quite similar for these heterogeneous algorithms, the difference becomes noticeable when decreasing the $I_{\mathrm{col}}$ values. In particular, for $I_{\mathrm{col}}=1 \mathrm{~s}$, Baseline drops down the RB utilization to $47 \%$, OFC to $37 \%$, OFCDS-Real to $27 \%$, while OFCDS-Ideal to $23 \%$. Notice that Baseline uses more resources than OFC and OFCDS, since it does not consider the CQI of the elected forwarders, meaning that these vehicles send more aggregated information while having a possibly very bad CQI, wasting much more resources. The same behavior can be observed for the other considered parameter configurations, but with overall less used RBs. It is also worth pointing out that increasing the load on the DSRC channel does not affect the LTE resource utilization, as can be seen from Figures $6 \mathrm{~b}$ and $6 \mathrm{c}$. 


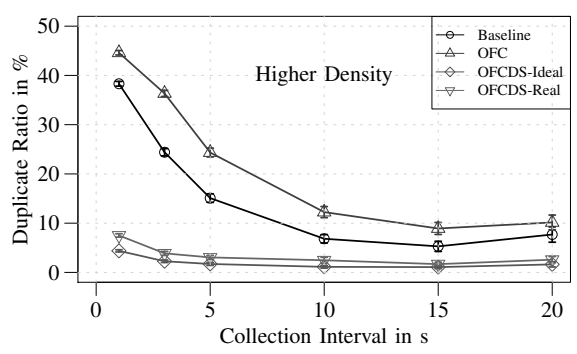

(a) $I_{\mathrm{LDB}}=1 \mathrm{~Hz}$ and $P^{\mathrm{tx}}=20 \mathrm{~mW}$.

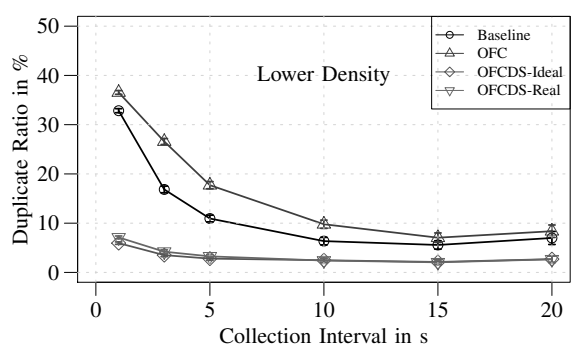

(b) $I_{\mathrm{LDB}}=1 \mathrm{~Hz}$ and $P^{\mathrm{tx}}=20 \mathrm{~mW}$.

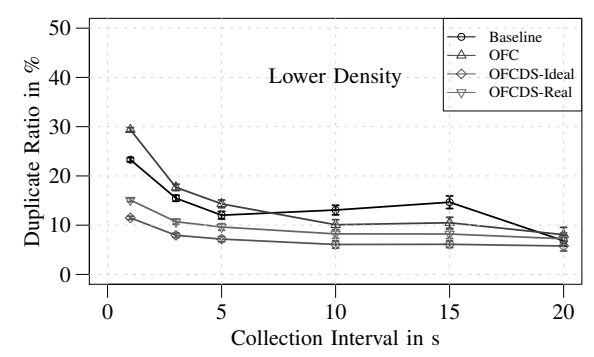

(c) $I_{\mathrm{LDB}}=10 \mathrm{~Hz}$ and $P^{\mathrm{tx}}=100 \mathrm{~mW}$.

Figure 7: The duplicates ratio as a function of the collection interval.

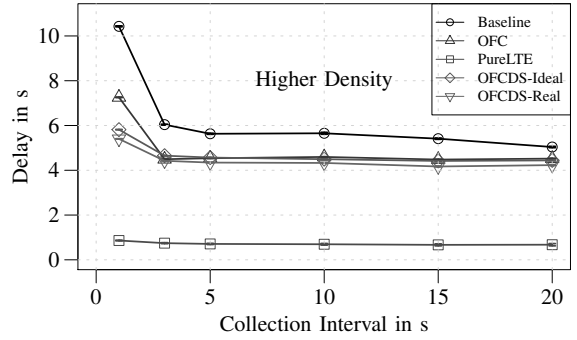

(a) $I_{\mathrm{LDB}}=1 \mathrm{~Hz}$ and $P^{\mathrm{tx}}=20 \mathrm{~mW}$.

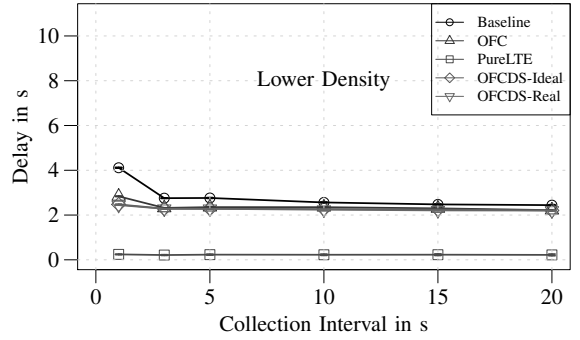

(b) $I_{\mathrm{LDB}}=1 \mathrm{~Hz}$ and $P^{\mathrm{tx}}=20 \mathrm{~mW}$.

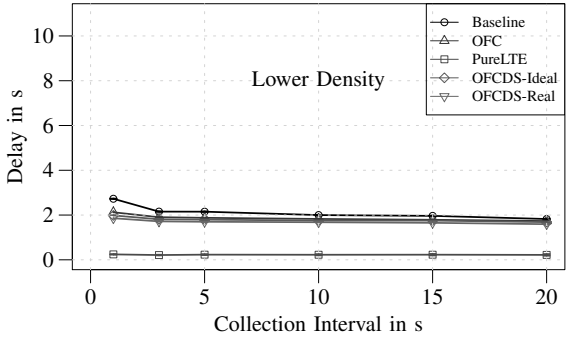

(c) $I_{\mathrm{LDB}}=10 \mathrm{~Hz}$ and $P^{\mathrm{tx}}=100 \mathrm{~mW}$.

Figure 8: Information delay as a function of the collection interval.

Figure 7 displays the mean duplicates ratio for different collection intervals. In general, all the considered heterogeneous algorithms introduce duplicate messages. This is related to the DSRC network topology and can be explained by the network assortativity phenomenon [34] from complex network theory, which implies that directly connected nodes (i.e, nodes in the same neighborhood) are likely to have similar degree levels. On the other hand, with PureLTE each vehicle is sending its own FCD without generating duplicate messages, which is why we do not display it here.

A first observation is that $\mathrm{OFC}$ and Baseline increase the duplicates ratio for lower collection intervals, meaning that more information is being sent to the server. This confirms the fact that their inhibition mechanism is less efficient for greater $I_{\mathrm{LDB}} / I_{\text {col }}$ ratios. Also, notice that Baseline induces generally less duplicates than OFC (e.g., roughly $45 \%$ duplicates generated by OFC and $39 \%$ by Baseline, when considering the higher density scenario and $I_{\mathrm{col}}=1 \mathrm{~s}$ ). This is because Baseline gives priority to vehicles having more DSRC neighbors in the forwarder selection process, thus minimizing the number of forwarding vehicles. But, since OFC tends to elect as forwarders those vehicles with a better CQI in the LTE uplink, it is still able to utilize less resources with respect to Baseline, as can be seen from Figure 6 . However, the greatest impact over the suppression of duplicates ratio is given by OFCDS. In particular, for the higher density scenario and the lowest collection interval, OFCDS-Ideal and OFCDS-Real generate only 4 and $7 \%$ duplicates correspondingly. The difference is less noticeable when we put more load on the DSRC channel (see
Figure $7 \mathrm{c}$ ), indicating the fact that the congestion slightly affects the performance of the duplicate suppression mechanism. The overall results, however, confirm the efficiency of the new proposed duplicate suppression scheme.

\subsection{Delay analysis}

An important aspect to be investigated is how much time an FCD message needs to reach the server from the moment when it is generated. In Figure 8 we compare the considered algorithms in terms of FCD message delay for different parameter configurations. We notice that the message transferring delay introduced by PureLTE is lower with respect to the considered heterogeneous approaches. This is mainly due to the fact that the size of the messages is smaller, since there is no aggregation (i.e., every vehicle is sending its own FCD message with a constant size). On the other hand, in case of a heterogeneous approach, the elected forwarders are sending aggregated messages, meaning that they send much more information, hence, needing more time to complete the transmission. Another reason is that in PureLTE the FCD message generation and the transmission starting time instances are the same, while in the heterogeneous algorithms the aggregated information to be sent via LTE has already an additional delay uniformly distributed between 0 and $I_{\mathrm{LDB}}$. However, it is worth noting that we measure here only the data transfer delay, without considering the random access procedure.

Among the heterogeneous approaches, Baseline has the highest message delay, especially for low collection intervals. There are two main reasons for this: the first one is that Baseline does not consider the CQI in the LTE uplink 


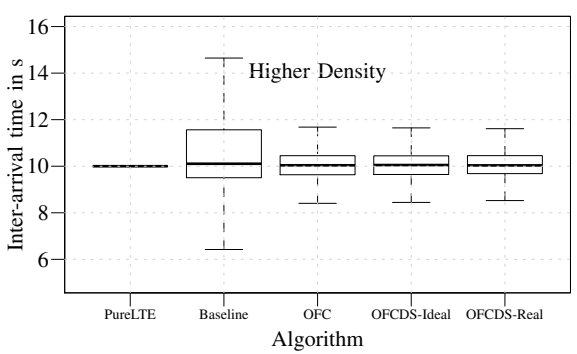

(a) $I_{\mathrm{LDB}}=1 \mathrm{~Hz}$ and $P^{\mathrm{tx}}=20 \mathrm{~mW}$.

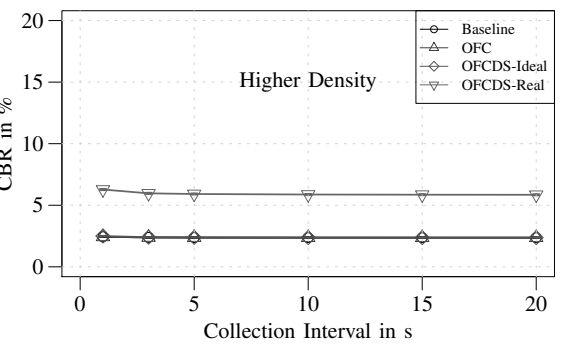

(a) $I_{\mathrm{LDB}}=1 \mathrm{~Hz}$ and $P^{\mathrm{tx}}=20 \mathrm{~mW}$.

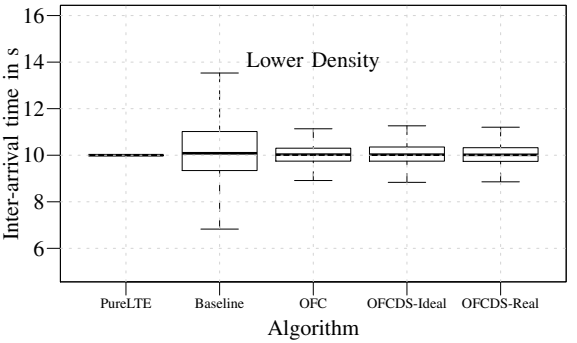

(b) $I_{\mathrm{LDB}}=1 \mathrm{~Hz}$ and $P^{\mathrm{tx}}=20 \mathrm{~mW}$.

Figure 9: Inter-arrival time for $I_{\mathrm{col}}=10 \mathrm{~s}$.

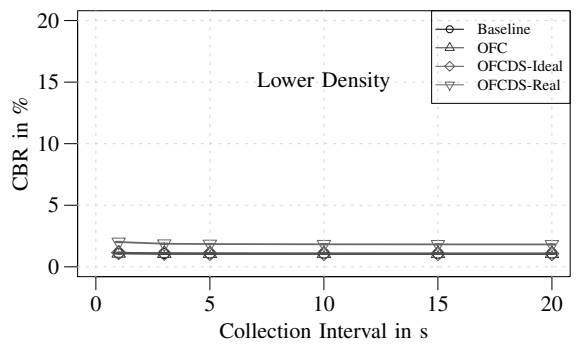

(b) $I_{\mathrm{LDB}}=1 \mathrm{~Hz}$ and $P^{\mathrm{tx}}=20 \mathrm{~mW}$.

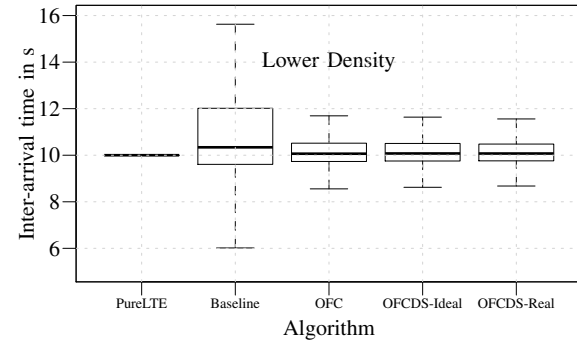

(c) $I_{\mathrm{LDB}}=10 \mathrm{~Hz}$ and $P^{\mathrm{tx}}=100 \mathrm{~mW}$.

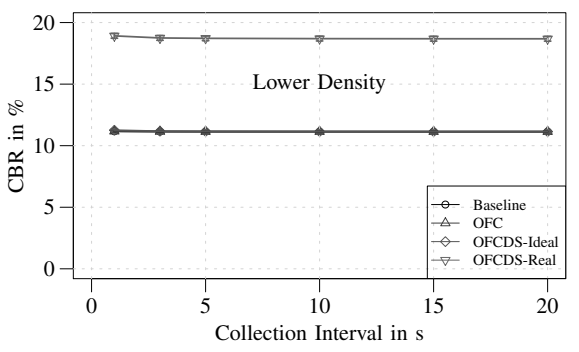

(c) $I_{\mathrm{LDB}}=10 \mathrm{~Hz}$ and $P^{\mathrm{tx}}=100 \mathrm{~mW}$.

Figure 10: The DSRC Channel Busy Ratio as a function of the collection interval.

when electing forwarding vehicles, which not only leads to a higher RB utilization, as we see in Figure 6 , but also to an increased transfer delay; the second reason is that Baseline is transferring more information due to the higher duplicates ratio (see Figure 7). The amount of the transferred information is precisely the reason why for the lower vehicular density scenario the average delays are generally smaller. Moreover, Figure $8 \mathrm{c}$ also suggests the fact that an increased load on the DSRC channel does not have any significant impact on the information delay. The delays induced by OFC and OFCDS are quite similar, with a slight difference for very low collection intervals, where the generated duplicates have a more negative impact on OFC with respect to OFCDS.

The FCD messages inter-arrival time is displayed in Figure 9 for a collection interval equal to $10 \mathrm{~s}$. The values are grouped in box plots, where the box itself is representing the first and third quartiles, the median value is represented by means of a central line inside each box, while the whiskers are showing the maximum and minimum values. We can notice that all the considered algorithms have roughly the same median value, which is equal to the requested collection interval. This means that the information is arriving at the remote server with the same frequency as it is requested. What changes is the distribution of the inter-arrival times. The best result is given by the PureLTE algorithm, since every vehicle is sending its own information at the requested update frequency (i.e., collection interval). The only variability here can come from the different message transferring delays. Although each vehicle is sending the same amount of information, the delay depends on the quality of the LTE channel. However, since the single FCD message size is constant and relatively small, this variability is not so visible here.

The story is slightly different for the heterogeneous algorithms. Here, besides the data packets transferring delays, the variability is also caused by the fact that the forwarding vehicles are re-elected at each collection period. Since a single vehicle's FCD message may be sent by different forwarders in different collection periods, we have an additional variability already at the sender side. This is visible when looking at OFC and OFCDS, whose results are similar among them, since the forwarder election mechanism is the same. The largest distribution of the inter-arrival time values is given by Baseline, because this algorithm does not consider the CQI in the LTE uplink, which leads to a higher variability due to the data transferring delays. The DSRC channel load also affects the inter-arrival time metric, as can be seen from Figure 9c, suggesting the fact that an increased load on the DSRC channel leads to a higher variability of the update beacons being received by a vehicle.

\subsection{Evaluation of the impact on DSRC}

In Figure 10 we show the impact that each of the considered heterogeneous algorithms has on the DSRC channel in terms of CBR as a function of the collection interval. An interesting observation is that, for all the considered parameter configurations, OFCDS-Real gives a higher CBR with respect to all other solutions, independently from the considered collection interval. These results are consistent with the fact that obtaining 2-hop DSRC awareness comes 
with a cost, especially if we attach to every beacon the raw current ID list of neighboring vehicles, as OFCDS-Real does. Notice that for the lower density scenario and beaconing frequency (Figure 10b) the overall CBR is smaller, since we have less vehicles periodically sending their beacons and less often. Same scenario but with 10 times the beaconing frequency (Figure 10c) leads to 10 times more load on the DSRC channel. OFCDS-Ideal has the same performance in terms of CBR as Baseline and OFC. This confirms the fact that the higher CBR induced by OFCDS-Real is caused only by the ID lists attached to the beacons. This also suggests that if we can come out with a good compression algorithm for the ID lists, we can do at most as well as OFCDS-Ideal.

It is worth noting that the CBR remains constant when varying the collection interval, with only a very small increase for $I_{\text {col }}=1 \mathrm{~s}$. This confirms the fact that beaconing is the main cause that affects the mean DSRC channel utilization, while the additional load induced by the Inhibit and Notify messages is insignificant. However, the mean CBR represented in Figure 10 is not uniformly distributed in time and/or space. As expected, we noticed that CBR depends on the current neighborhood vehicular density, meaning that at an individual level, each vehicle experiences a CBR that depends on the current number of DSRC neighbors. In time, we noticed that vehicles roaming in crowded neighborhoods experience periodic spikes of CBR, which are observed when Inhibit and Notify messages are broadcasted. On the downside, these control messages are a direct consequence of vehicles being elected as forwarders, meaning that the spikes are caused by our proposed algorithm. On the upside, their impact on the overall system, and specifically on the mean CBR, is insignificant, as can be seen in Figure 10. Also, the algorithm itself is something that we can control, meaning that smoothing out these spikes can be the subject of a future work.

\section{Conclusion}

In this paper we address the problem of intermittent Floating Car Data collection in an urban environment by exploiting both DSRC and LTE technologies. We propose OFCDS, an on-the-fly distributed clustering algorithm with an efficient duplicate suppression mechanism. The main features of our proposed solution are: (i) it relies on a distributed procedure to periodically select forwarding vehicles in charge of sending their own data, as well as their neighboring vehicles' information, towards a remote facility via LTE; (ii) the forwarder selection process is based on timers that depend on parameters drawn from both DSRC and LTE communication technologies; (iii) it exploits the existing background beaconing process to populate a local database used by the forwarding vehicles to create the aggregated information to be sent via LTE; (iv) it uses additional control messages sent on DSRC to suppress the generated duplicates.
The efficiency of the proposed algorithm is proved by means of an extensive performance analysis based on realistic simulations. In particular, we show that a proper cooperation between the VANET based on the DSRC technology and the LTE cellular network brings a significant benefit in terms of LTE radio resources utilization. The price that we have to pay for offloading the LTE access network consists in an increase of the data transferring delay. However, this delay might be compensated by a significantly lower number of vehicles simultaneously competing during the random access procedure.

\section{References}

[1] M. S. Ali, E. Hossain, D. I. Kim, LTE/LTE-A Random Access for Massive Machine-Type Communications in Smart Cities, IEEE Communications Magazine 55 (1) (2017) 76-83. doi:10.1109/MCOM.2017.1600215CM.

[2] E. Soltanmohammadi, K. Ghavami, M. Naraghi-Pour, A Survey of Traffic Issues in Machine-to-Machine Communications Over LTE, IEEE Internet of Things Journal 3 (6) (2016) 865-884. doi:10.1109/JIOT.2016.2533541.

[3] G. C. Madueño, J. J. Nielsen, D. M. Kim, N. K. Pratas, Č. Stefanović, P. Popovski, Assessment of LTE Wireless Access for Monitoring of Energy Distribution in the Smart Grid, IEEE Journal on Selected Areas in Communications 34 (3) (2016) 675-688. doi:10.1109/JSAC.2016.2525639.

[4] T. P. C. de Andrade, C. A. Astudillo, L. R. Sekijima, N. L. S. da Fonseca, The Random Access Procedure in Long Term Evolution Networks for the Internet of Things, IEEE Communications Magazine 55 (3) (2017) 124-131. doi:10.1109/MCOM.2017.1600555CM.

[5] C. Sommer, F. Dressler, Vehicular Networking, Cambridge University Press, 2014. doi:10.1017/CBO9781107110649.

[6] O. Altintas, F. Dressler, F. Hagenauer, M. Matsumoto, M. Sepulcre, C. Sommer, Making Cars a Main ICT Resource in Smart Cities, in: 34th IEEE Conference on Computer Communications (INFOCOM 2015), International Workshop on Smart Cities and Urban Informatics (SmartCity 2015), IEEE, Hong Kong, China, 2015, pp. 654-659. doi:10.1109/INFCOMW.2015.7179448.

[7] G. Araniti, C. Campolo, M. Condoluci, A. Iera, A. Molinaro, LTE for Vehicular Networking: A Survey, IEEE Communications Magazine $51 \quad$ (5) (2013) 148-157. doi:10.1109/MCOM.2013.6515060.

[8] R. N. Clarke, Expanding mobile wireless capacity: The challenges presented by technology and economics, Telecommunications Policy $38 \quad(8-9) \quad$ (2014) 693-708. doi:10.1016/j.telpol.2013.11.006.

[9] J. Barrachina, P. Garrido, M. Fogue, F. J. Martinez, J.C. Cano, C. T. Calafate, P. Manzoni, Road Side Unit Deployment: A Density-Based Approach, IEEE Intelligent Transportation Systems Magazine 5 (3) (2013) 30-39. doi:10.1109/mits.2013.2253159.

[10] I. Turcanu, C. Sommer, A. Baiocchi, F. Dressler, Pick the Right Guy: CQI-Based LTE Forwarder Selection in VANETs, in: 8th IEEE Vehicular Networking Conference (VNC 2016), IEEE, Columbus, OH, 2016, pp. 98-105. doi:10.1109/VNC.2016.7835938.

[11] ETSI, Intelligent Transport Systems (ITS); Vehicular Communications; Basic Set of Applications; Part 2: Specification of Cooperative Awareness Basic Service, Tech. Rep. 302 637-2 V1.3.2, ETSI (Nov. 2014).

[12] ETSI, Intelligent Transport Systems (ITS); Vehicular Communications; Basic Set of Applications; Local Dynamic Map (LDM), EN 302895 V1.1.1, ETSI (Sep. 2014).

[13] T. Mangel, T. Kosch, H. Hartenstein, A comparison of UMTS and LTE for Vehicular Safety Communication at Intersections, in: 2nd IEEE Vehicular Networking Conference 
(VNC 2010), IEEE, Jersey City, NJ, 2010, pp. 293-300. doi:10.1109/VNC.2010.5698244.

[14] C. Ide, B. Dusza, M. Putzke, C. Wietfeld, Channel sensitive transmission scheme for V2I-based Floating Car Data collection via LTE, in: IEEE International Conference on Communications (ICC 2012), IEEE, Ottawa, Canada, 2012, pp. 7151-7156. doi:10.1109/ICC.2012.6364684.

[15] SAE, DSRC Message Communication Minimum Performance Requirements: Basic Safety Message for Vehicle Safety Applications, Draft Std. J2945.1 Revision 2.2, SAE (Apr. 2011).

[16] C. Ide, F. Kurtz, C. Wietfeld, Cluster-Based Vehicular Data Collection for Efficient LTE Machine-Type Communication, in: 78th IEEE Vehicular Technology Conference Fall (VTC2013-Fall), IEEE, Las Vegas, NV, 2013, pp. 1-5. doi:10.1109/VTCFall.2013.6692136.

[17] R. Stanica, M. Fiore, F. Malandrino, Offloading Floating Car Data, in: 14th IEEE International Symposium on a World of Wireless, Mobile and Multimedia Networks (WoWMoM 2013), IEEE, Madrid, Spain, 2013, pp. 1-9. doi:10.1109/WoWMoM.2013.6583391.

[18] S. Ancona, R. Stanica, M. Fiore, Performance boundaries of massive Floating Car Data offloading, in: 11th Annual Conference on Wireless On-demand Network Systems and Services (WONS 2014), IEEE, Obergurgl, Austria, 2014, pp. 89-96. doi:10.1109/WONS.2014.6814727.

[19] A. Benslimane, T. Taleb, R. Sivaraj, Dynamic ClusteringBased Adaptive Mobile Gateway Management in Integrated VANET - 3G Heterogeneous Wireless Networks, IEEE Journal on Selected Areas in Communications 29 (3) (2011) 559-570. doi:10.1109/JSAC.2011.110306.

[20] G. El Mouna Zhioua, N. Tabbane, H. Labiod, S. Tabbane, A Fuzzy Multi-Metric QoS-Balancing Gateway Selection Algorithm in a Clustered VANET to LTE Advanced Hybrid Cellular Network, IEEE Transactions on Vehicular Technology 64 (2) (2015) 804-817. doi:10.1109/TVT.2014.2323693.

[21] G. Remy, S.-M. Senouci, F. Jan, Y. Gourhant, LTE4V2XCollection, dissemination and multi-hop forwarding, in: IEEE International Conference on Communications (ICC), 2012, IEEE, Nanjing, Jiangsu, 2012, pp. 120-125. doi:10.1109/ICC.2012.6364412.

[22] A. Bazzi, B. M. Masini, G. Pasolini, V2V and V2R for cellular resources saving in vehicular applications, in: IEEE Wireless Communications and Networking Conference (WCNC 2012), IEEE, Paris, France, 2012, pp. 3199-3203. doi:10.1109/WCNC.2012.6214358.

[23] R. S. Bali, N. Kumar, J. J. Rodrigues, Clustering in vehicular ad hoc networks: Taxonomy, challenges and solutions, Elsevier Vehicular Communications 1 (3) (2014) 134-152. doi:10.1016/j.vehcom.2014.05.004.

[24] ETSI, LTE E-UTRA Physical layer procedures (3GPP TS 36.213), TS 136213 V13.2.0, ETSI (Aug. 2016).

[25] E. Dahlman, S. Parkvall, J. Sköld, 4G LTE / LTE-Advanced for Mobile Broadband, Academic Press, 2014.

[26] A. Virdis, G. Stea, G. Nardini, SimuLTE - A Modular Systemlevel Simulator for LTE/LTE-A Networks based on OMNeT++, in: 4th International Conference on Simulation and Modeling Methodologies, Technologies and Applications (SIMULTECH 2014), Vienna, 2014, pp. 59-70. doi:10.5220/0005040000590070.

[27] I. T. Union, Guidelines for evaluation of radio interface technologies for IMT-Advanced, Report M.2135-1, ITU-R (Dec. 2009).

[28] S. Uppoor, M. Fiore, Characterizing Pervasive Vehicular Access to the Cellular RAN Infrastructure: An Urban Case Study, IEEE Transactions on Vehicular Technology 64 (6) (2015) 2603-2614. doi:10.1109/TVT.2014.2343651.

[29] ETSI, Intelligent Transport Systems (ITS); Decentralized Congestion Control Mechanisms for Intelligent Transport Systems operating in the $5 \mathrm{GHz}$ range; Access layer part, TS 102687 V1.1.1, ETSI (Jul. 2011).

[30] C. Sommer, O. K. Tonguz, F. Dressler, Adaptive Beaconing for Delay-Sensitive and Congestion-Aware Traffic Information Systems, in: 2nd IEEE Vehicular Networking Con- ference (VNC 2010), IEEE, Jersey City, NJ, 2010, pp. 1-8. doi:10.1109/VNC.2010.5698242.

[31] F. Hagenauer, F. Dressler, C. Sommer, A Simulator for Heterogeneous Vehicular Networks, in: 6th IEEE Vehicular Networking Conference (VNC 2014), Poster Session, IEEE, Paderborn, Germany, 2014, pp. 185-186. doi:10.1109/VNC.2014.7013339.

[32] C. Sommer, R. German, F. Dressler, Bidirectionally Coupled Network and Road Traffic Simulation for Improved IVC Analysis, IEEE Transactions on Mobile Computing 10 (1) (2011) 3-15. doi:10.1109/TMC.2010.133.

[33] C. Sommer, D. Eckhoff, R. German, F. Dressler, A Computationally Inexpensive Empirical Model of IEEE 802.11p Radio Shadowing in Urban Environments, in: 8th IEEE/IFIP Conference on Wireless On demand Network Systems and Services (WONS 2011), IEEE, Bardonecchia, Italy, 2011, pp. 84-90. doi:10.1109/WONS.2011.5720204.

[34] S. Boccaletti, V. Latora, Y. Moreno, M. Chavez, D.U. Hwang, Complex networks: Structure and dynamics, Elsevier Physics Reports 424 (4-5) (2006) 175-308. doi:10.1016/j.physrep.2005.10.009. 